\title{
Ibutilide-Induced Alterations in Electrocardiographic and Spatial Vectorcardiographic Descriptors of Ventricular Repolarization
}

\author{
Polychronis Dilaveris, M.D., Fesc, Athanasios Theoharis, M.D., Petros GiaOuris, M.D., Aristides Anastasopoulos, M.D., \\ Bruno Zumerle, M.D., George Tzannetis, M.D., Christodoulos SteFanadis, M.D., FeSC, FaCC* \\ Department of Cardiology, Pammakaristos Hospital, and the *University Department of Cardiology, Hippokration Hospital, \\ Athens, Greece
}

\section{Summary}

Background: Ibutilide is used for the pharmacologic cardioversion of atrial fibrillation (AF) or flutter (AFl). Ibutilideinduced QT interval prolongation has been demonstrated previously. However, its effects on vectorcardiographic (VCG) descriptors of ventricular repolarization (VR) have not been studied so far.

Hypothesis: To evaluate the effects of ibutilide on electrocardiographic (ECG) and VCG descriptors of VR, one or two repeated 10-min infusions of $1 \mathrm{mg}$ of ibutilide were given in 50 consecutively recruited patients (36 women, mean age 69.4 \pm 9.3 years) with $\mathrm{AF}$ or $\mathrm{AFl}$ of recent onset.

Methods: The maximum and the minimum QT intervals, QT dispersion, the rate-corrected QT maximum, and the spatial VCG descriptors, spatial T amplitude, and spatial QRS-T angle were calculated before (baseline ECG) and $30 \mathrm{~min}$ after the start of ibutilide infusion (postinfusion ECG).

Results: After ibutilide infusion, 40 (80\%) patients were cardioverted to sinus rhythm (Group 1), while in the remaining 10 (Group 2) AF or AFl persisted. In both study groups, temporal measures of VR were significantly increased from baseline to the postinfusion ECG. In Group 1, spatial T amplitude and spatial QRS-T angle did not differ between those two ECGs, while in Group 2 spatial T amplitude was significantly increased $(\mathrm{p}=0.005)$ and spatial QRS-T angle was significantly decreased $(\mathrm{p}=0.002)$ post infusion compared with baseline ECG.

Conclusions: While temporal measures of VR are significantly affected in all patients who receive ibutilide infusion for AF or AFl cardioversion, spatial VCG descriptors of VR are

Address for reprints:

Polychronis E. Dilaveris, M.D., FESC

22 Miltiadou Str.

GR-15561 Holargos

Athens, Greece

e-mail: hrodil@yahoo.com

Received: May 6, 2003

Accepted with revision: September 8, 2003 significantly altered only in those patients who fail to respond to the drug. A dose-related effect of ibutilide on the different aspects of VR should be suspected.

Key words: ventricular repolarization, electrocardiogram, vectorcardiogram

\section{Introduction}

Ibutilide is a pure class III antiarrhythmic agent that acts predominantly by prolonging myocardial action potential duration. ${ }^{1}$ It is considered to be a useful agent for pharmacologic cardioversion of recent-onset atrial fibrillation (AF) or flutter (AFl). ${ }^{1}$ Ibutilide significantly prolonged the rate-corrected QT (QTc) interval in electrocardiographic (ECG) studies in healthy volunteers and patients with $\mathrm{AF}$ or $\mathrm{AFl}{ }^{2-5}$ This effect was dose related and correlated directly with plasma ibutilide concentrations. ${ }^{3}$ The QTc interval reflects the duration of repolarization of the ventricles, and its prolongation is indicative of class III antiarrhythmic activity. Several studies have lately focused on the spatial T-loop morphology features as an accurate measure of repolarization heterogeneity. ${ }^{6-11}$ The effects of ibutilide infusion on these spatial vectorcardiographic (VCG) descriptors of ventricular repolarization (VR) have not been studied so far.

The objective of the present study was to evaluate the effects of ibutilide on ECG and VCG descriptors of VR in patients with $\mathrm{AF}$ or $\mathrm{AFl}$ of recent onset.

\section{Methods}

\section{Study Population}

The study population consisted of 50 consecutively recruited patients (36 women, mean age $69.4 \pm 9.3$ years) admitted to the hospital because of a symptomatic episode of AF or AFl of recent onset ( $<2$ days). All patients underwent physical examination, 12-lead ECG, and serial blood tests. No patient had clinically overt heart failure (New York Heart Association [NYHA] classes II-IV) or history of a recent ( $<1$ month) previous myocardial infarction. No patient showed severe $(<50$ beats/min) bradycardia on admission ECG. Serum potassium levels were $>4 \mathrm{mEq} / \mathrm{l}$ in all patients. Routine medications were 
not withheld during patient evaluation. Excluded from the study were patients with left or right bundle-branch block, atrioventricular block, ventricular preexcitation, prior pacemaker implantation, or pericarditis. Patients receiving digitalis or any drugs known to influence the QT interval were also excluded.

Ibutilide fumarate $1 \mathrm{mg}$ was given in a 10-min slow intravenous infusion in all 50 patients. The same dose was repeated 10 min later in case the arrhythmia persisted. Ibutilide infusion was stopped in the case of arrhythmia conversion to sinus rhythm or if significant proarrhythmia ensued. Drug conversion was considered successful when sinus rhythm was achieved within the first $60 \mathrm{~min}$ after the start of ibutilide infusion. All patients were continuously monitored during ibutilide infusion and for $4 \mathrm{~h}$ thereafter. Digital 12-lead resting ECGs were recorded in all patients immediately before the start of ibutilide administration (baseline ECG) and 30 min later (post infusion ECG).

\section{Twelve-Lead Surface Electrocardiogram}

In all subjects, a 12-lead digital ECG was recorded in supine resting position (Fig. 1A) using a computer-basedECG

(A)
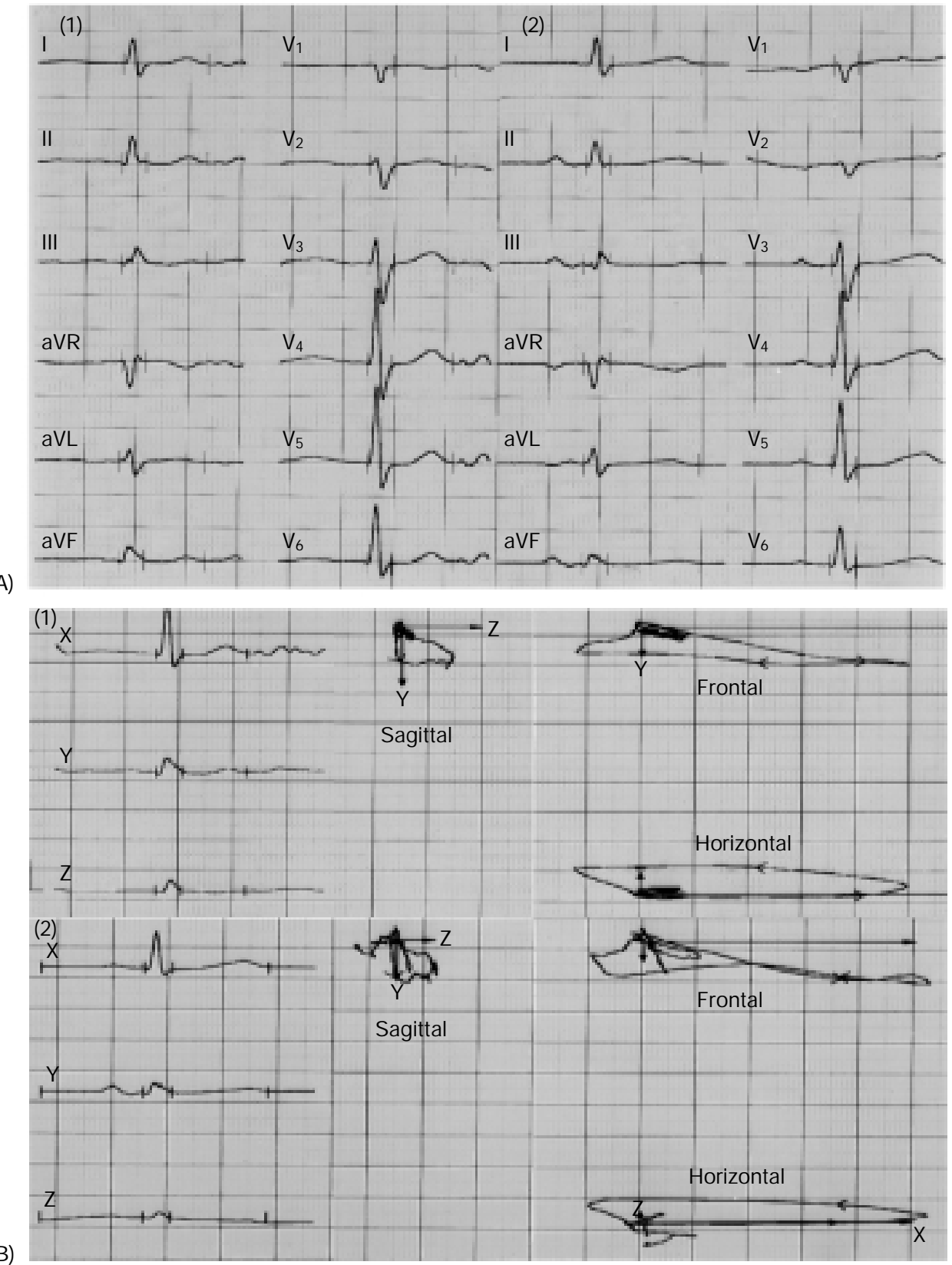

FIG. 1 (A) Twelve-lead electrocardiogram. (B) Reconstructed X, Y, and Z leads and P, QRS and T-loop projections in the frontal, horizontal, and right sagittal planes from a 76-year-old patient before (1) and after (2) cardioversion to sinus rhythm. 
machine (Schiller AT2 Plus, Schiller AG Corp., Baar, Switzerland). All 12 leads of each ECG were recorded simultaneously for $10 \mathrm{~s}$ and sampled at a rate of $1000 \mathrm{~Hz}$. From each lead, the average complex was automatically calculated (SEMA2001.81 software, Schiller AG). All QT interval measurements were automatically calculated by our analysis system (SEMA-200 1.81 software, Schiller AG). The QT interval was measured from the QRS onset to the end of the $\mathrm{T}$ wave. The point of T-wave offset was defined as the return to baseline. ${ }^{12}$ Maximum and minimum QT intervals and QT dispersion (QT maximum - QT minimum) were calculated from each ECG; QT maximum was corrected for heart rate using Bazett's formula $(\mathrm{QTc}$ maximum $=\mathrm{QT}$ maximum/RR interval).

\section{Twelve-Lead Vectorcardiogram}

To derive VCG descriptors of VR, orthogonal X, Y, and Z leads were reconstructed from the standard 12 ECG leads (Fig. 1B).$^{13}$ Let QRSx, QRSy, and QRSz be the projections of the maximum $\mathrm{QRS}$ vector on the $\mathrm{X}, \mathrm{Y}$, and $\mathrm{Z}$ axes, and $\mathrm{Tx}$, $T y$, and Tz be the projections of the maximum $T$ vector on the $\mathrm{X}, \mathrm{Y}$, and Z axes, respectively. QRSx, QRSy, QRSz, Tx, Ty, and Tz were automatically calculated by our analysis system. According to previously published equations, ${ }^{14}$ we calculated the amplitude of the maximum spatial $\mathrm{T}$ vector (spatial $\mathrm{T}$ amplitude) from the formula:

$$
\text { spatial } \mathrm{T} \text { amplitude }=\left(\mathrm{Tx}^{2}+\mathrm{Ty}^{2}+\mathrm{Tz}^{2}\right)^{1 / 2}
$$

and the angle $\left(\theta^{\circ}\right)$ between the maximum spatial QRS and T vectors (spatial QRS-T angle) from the formula: ${ }^{14}$

$$
\cos \theta^{\circ}=(\mathrm{QRSxTx}+\mathrm{QRSyTy}+\mathrm{QRSzTz}) /|\mathrm{QRS}||\mathrm{T}|
$$

\section{Statistical Analysis}

Continuous variables are expressed as mean \pm standard deviation. Mann-Whitney, Wilcoxon, and chi-square tests were used where appropriate for between-group comparisons. $P$ values $<0.05$ were considered statistically significant.

\section{Results}

Of a total enrollment of 50 patients with $\mathrm{AF}$ or $\mathrm{AFl}, 40$ $(80 \%)$ were cardioverted to sinus rhythm within the first hour after the start of ibutilide infusion (Group 1), while in the remaining 10 (Group 2), AF or AFl persisted for the total 4-h monitoring period after the termination of ibutilide infusion. The clinical characteristics of these two study groups are listed in Table I. There were no significant differences in demographic or clinical characteristics between the two groups. Only gender and duration of the last episode of AF or AFl differed between the two groups. The latter was higher in Group 2 than in Group 1 patients (Table I). The total dose of ibutilide given was significantly higher in Group 2 than in Group 1 patients (2 vs. $1.2 \pm 0.4 \mathrm{mg}$, respectively, $\mathrm{p}<0.0001)$. No significant proarrhythmia ensued during ibutilide infusion or in the fol-
TABLE I Clinical characteristics of patients who were (Group 1)

\begin{tabular}{|c|c|c|c|}
\hline Characteristic & $\begin{array}{l}\text { Group 1 } \\
(\mathrm{n}=40)\end{array}$ & $\begin{array}{c}\text { Group } 2 \\
(\mathrm{n}=10)\end{array}$ & $\mathrm{p}$ Value \\
\hline Age (years) & $70.5 \pm 5.8$ & $65 \pm 17.4$ & 0.574 \\
\hline Males, n (\%) & $8(20)$ & $6(60)$ & $<0.0001$ \\
\hline Previous episodes (n) & $2.1 \pm 2.1$ & $1.2 \pm 0.4$ & 0.395 \\
\hline Episodes/year (n) & $1.6 \pm 2.3$ & $0.4 \pm 0.5$ & 0.451 \\
\hline Duration of the last episode (h) & $8.7 \pm 6.9$ & $30.2 \pm 19.8$ & 0.004 \\
\hline $\operatorname{LAD}(\mathrm{mm})$ & $39 \pm 3.9$ & $38 \pm 1.6$ & 0.635 \\
\hline $\operatorname{LVEF}(\%)$ & $56.8 \pm 4.9$ & $57.5 \pm 2.6$ & 0.831 \\
\hline $\operatorname{LVEDD}(\mathrm{mm})$ & $51.4 \pm 3.6$ & $53 \pm 5.2$ & 0.467 \\
\hline \multicolumn{4}{|l|}{ Disease } \\
\hline Hypertension (\%) & $20(50)$ & $8(80)$ & \\
\hline Coronary artery disease $(\%)$ & $6(15)$ & 0 & \\
\hline Valvular heart disease (\%) & 0 & $2(20)$ & \\
\hline $\operatorname{COPD}(\%)$ & $4(10)$ & 0 & \\
\hline Other $(\%)$ & $2(5)$ & 0 & \\
\hline Lone $(\%)$ & $8(20)$ & 0 & \\
\hline \multicolumn{4}{|l|}{ Medications } \\
\hline ACE inhibitors (\%) & $14(35)$ & $6(60)$ & \\
\hline Calcium blockers (\%) & $10(25)$ & 0 & \\
\hline Beta blockers (\%) & $2(5)$ & 0 & \\
\hline Nitrates (\%) & $4(10)$ & 0 & \\
\hline Diuretics (\%) & $10(25)$ & 0 & \\
\hline
\end{tabular}
and who were not cardioverted to sinus rhythm (Group 2)

Abbreviations: $\mathrm{ACE}=$ angiotensin-converting enzyme, $\mathrm{COPD}=$ chronic obstructive pulmonary disease, $\mathrm{LAD}=$ left atrial maximal diameter, $\mathrm{LVEDD}=$ left ventricular end-diastolic diameter, $\mathrm{LVEF}=$ left ventricular ejection fraction.

lowing 4-h monitoring period. Heart rate, RR interval, maximum and the minimum QT interval, and QT dispersion were significantly changed from the baseline to the postinfusion ECG in Group 1 patients, while maximum QTc interval, spatial T amplitude, and spatial QRS-T angle did not differ between the two ECGs (Table II). In Group 2 patients, maximum QT, minimum QT, and maximum QTc intervals were significantly increased from baseline to the postinfusion ECG. Furthermore, spatial $\mathrm{T}$ amplitude was significantly increased and the spatial QRS-T angle was significantly decreased post infusion compared with baseline ECG (Table III).

Heart rate was marginally higher in Group 2 than in Group 1 patients in the baseline ECG $(p=0.04)$, while it was significantly higher in Group 2 than in Group 1 in the post-infusion ECG ( $\mathrm{p}<0.0001)$. QRS duration did not differ between the two patient groups, neither in baseline $(p=0.471)$, nor in postinfusion ECG $(\mathrm{p}=0.392)$. The differences in maximum QT, minimum QT, maximum QTc interval, and QT dispersion between the two study groups are shown for the baseline ECG in Figure 2 and for the postinfusion ECG in Figure 3. Spatial T amplitude and spatial QRS-T angle did not differ between the two study groups, neither in the baseline ECG $(\mathrm{p}=0.254$ and $\mathrm{p}$ $=0.473$, respectively $)$ nor in the postinfusion $\mathrm{ECG}(\mathrm{p}=0.236$ and $\mathrm{p}=0.326$, respectively). Spatial T amplitude and spatial QRS-T angle did not differ in the postinfusion ECG between 
TABLE II Changes in electrocardiographic and vectorcardiographic parameters from baseline to postinfusion electrocardiogram (ECG) in patients successfully cardioverted to sinus rhythm

\begin{tabular}{lccc}
\hline & Baseline & Postinfusion & \\
Parameter & ECG & ECG & p Value \\
\hline Heart rate (beats/min) & $120.7 \pm 26.2$ & $68.8 \pm 13$ & $<0.0001$ \\
RR interval (ms) & $522.1 \pm 125.6$ & $899.8 \pm 161$ & $<0.0001$ \\
QRS duration (ms) & $89.4 \pm 19.3$ & $89.6 \pm 18.6$ & 0.917 \\
Maximum QT (ms) & $359.5 \pm 35.9$ & $472.2 \pm 49.3$ & $<0.0001$ \\
Minimum QT (ms) & $315.2 \pm 42.7$ & $412.4 \pm 59.5$ & $<0.0001$ \\
QT dispersion $(\mathrm{ms})$ & $44.3 \pm 20.6$ & $59.8 \pm 22.7$ & 0.002 \\
Maximum QTc $(\mathrm{ms})$ & $502.2 \pm 36.2$ & $499.4 \pm 36.2$ & 0.716 \\
Spatial Tamplitude $(\mu \mathrm{V})$ & $267.7 \pm 129.3$ & $254 \pm 135.2$ & 0.458 \\
Spatial QRS-T angle & & & \\
$\quad$ (degrees) & $24.8 \pm 13.4$ & $23.7 \pm 15.1$ & 0.624 \\
\hline
\end{tabular}

See text for definitions of the individual indices.

those patients who received $1 \mathrm{mg}$ and those who received $2 \mathrm{mg}$ of ibutilide $(253.8 \pm 149.2$ vs. $286.7 \pm 116.6 \mu \mathrm{V}, \mathrm{p}=0.373$, and $24.6 \pm 16$ vs. $19 \pm 8.4$ degrees, $p=0.136$, respectively), although a trend toward higher spatial T-amplitude values and lower spatial QRS-T-angle values was noted in those patients who received $2 \mathrm{mg}$ of ibutilide fumarate.

\section{Discussion}

The present study demonstrated the effects of ibutilide infusion on ECG and VCG descriptors of VR in patients with AF or AFl episodes of recent onset. While ECG measures of VR were significantly affected in all patients who received ibutilide infusion, spatial VCG descriptors of VR were significantly altered only in those patients who failed to respond to the drug and to be cardioverted to sinus rhythm. A dose-related effect of ibutilide infusion on the different aspects of VR should be suspected.

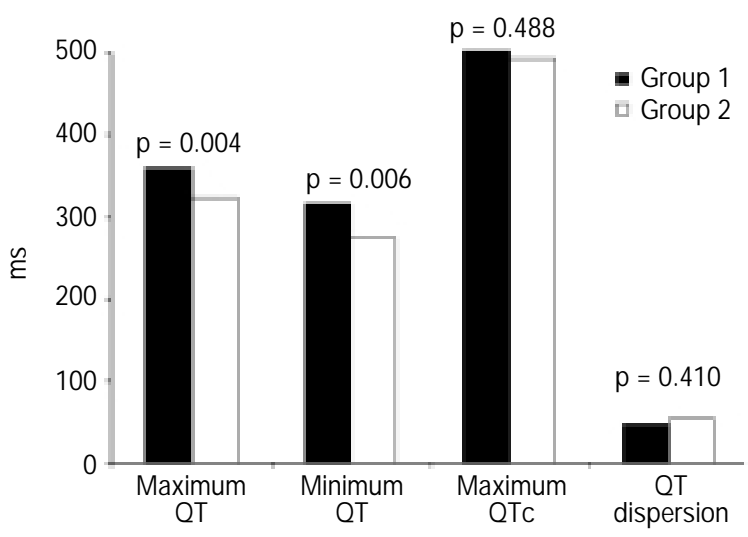

FIG. 2 Electrocardiographic parameters in patients successfully cardioverted to sinus rhythm (Group 1) and those who failed to respond to ibutilide infusion (Group 2) in the baseline ECG.
TABLE III Changes in electrocardiographic and vectorcardiographic parameters from baseline to postinfusion electrocardiogram (ECG) in patients who failed to be cardioverted to sinus rhythm

\begin{tabular}{lccr}
\hline & Baseline & Postinfusion & \\
Parameter & ECG & ECG & p Value \\
\hline Heart rate (beat/min) & $139.2 \pm 16.3$ & $124.2 \pm 11.1$ & 0.054 \\
RR interval (ms) & $436 \pm 51.3$ & $486.2 \pm 42.6$ & 0.054 \\
QRS duration (ms) & $84.8 \pm 9$ & $84.4 \pm 6.1$ & 0.858 \\
Maximum QT (ms) & $324.4 \pm 8.7$ & $371.6 \pm 34.8$ & 0.003 \\
Minimum QT (ms) & $273.2 \pm 32$ & $323.2 \pm 55.5$ & 0.004 \\
QT dispersion $(\mathrm{ms})$ & $51.2 \pm 33$ & $48.4 \pm 27.1$ & 0.817 \\
Maximum QTc $(\mathrm{ms})$ & $493.2 \pm 37.1$ & $531.8 \pm 28.6$ & 0.038 \\
Spatial T amplitude $(\mu \mathrm{V})$ & $215.6 \pm 119.9$ & $312.4 \pm 146.4$ & 0.005 \\
Spatial QRS-T angle & & & \\
(degrees) & $28.4 \pm 14.6$ & $18.8 \pm 8.8$ & 0.002 \\
\hline
\end{tabular}

See text for definitions of the individual indices.

\section{Ibutilide-Induced Repolarization Alterations}

Ibutilide is an antiarrhythmic agent with minimal hemodynamic, but major electrophysiologic effects that lead to its characterization as a class III agent. It lengthens the effective refractory period in both the atrium and the ventricle while having little effect on conduction in normal cardiac tissue. It prolongs the action potential duration and effective refractory periods in both the atria and the ventricles. ${ }^{1-5}$ In previous studies, ibutilide significantly prolonged the QTc interval in ECG studies in healthy volunteers and patients with $\mathrm{AF}$ or $\mathrm{AFl} .^{2-5}$ This effect was dose related and correlated directly with plasma ibutilide concentrations. ${ }^{3}$

In this study, although QT maximum, QT minimum, and QT dispersion significantly increased from baseline to the postinfusion ECG in Group 1 patients, QTc maximum failed to demonstrate significant changes from the first to the second ECG. Therefore, the alterations in QT uncorrected values

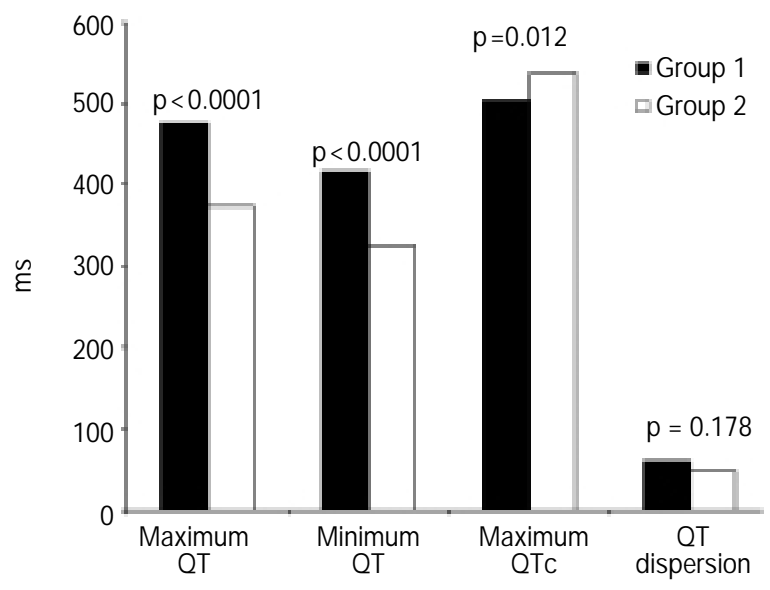

FIG. 3 Electrocardiographic parameters in patients successfully cardioverted to sinus rhythm (Group 1) and those who failed to respond to ibutilide infusion (Group 2) in the postinfusion ECG. 
should rather be attributed to heart rate changes from baseline to the postinfusion ECG. In Group 2 patients, who received a higher ibutilide dose than did Group 1 patients, both uncorrected and heart rate-corrected QT interval values showed a significant increase from baseline to the postinfusion ECG. In this case, QT interval alterations during ibutilide infusion may be attributed not only to heart rate changes but also to VR alterations during ibutilide infusion. These findings are in contrast to previous publications, which showed comparable effects of ibutilide infusion on both uncorrected and heart rate-corrected QT interval values. Only in Group 2 patients, who received a larger ibutilide dose, were our results comparable with those previously reported. ${ }^{5}$ The dose-related effect of ibutilide infusion on VR is further verified from the preferential effect of ibutilide infusion on the spatial VCG characteristics of VR only in Group 2 patients who received a greater ibutilide dose, but not in Group 1 patients. When VCG descriptors that were obtained from the postinfusion ECG were analyzed according to the dose of ibutilide given, a trend toward higher spatial T-amplitude values and lower spatial QRS-T angle values was noted only in those patients who received $2 \mathrm{mg}$ compared with those who received only $1 \mathrm{mg}$ of ibutilide. Therefore, the underlying heart rhythm as well as heart rate should also be considered as possible contributing factors to the alterations of the VCG descriptors of VR that are obtained after ibutilide infusion. Furthermore, we cannot exclude the impact of the underlying heart disease and the concomitant medications already administered in our study patients on the observed differences in the ECG or VCG descriptors of VR between the two study groups.

This is the first study to demonstrate the effects of ibutilide infusion on the spatial VCG descriptors of VR. The spatial T amplitude was significantly increased from baseline to the postinfusion ECG, indicating the presence of globally taller $\mathrm{T}$ waves after ibutilide infusion than previously. On the other hand, the spatial QRS-T angle, which is considered a marker of the repolarization heterogeneity, ${ }^{6-8}$ was significantly decreased from baseline to the postinfusion ECG. These ibutilide-induced alterations on the VCG descriptors of VR were evident only in Group 2 patients who received a larger ibutilide dose than did Group 1 patients. We may assume that a high ibutilide dose prolongs VR in a more or less global and homogeneous fashion. None of our patients demonstrated ibutilideinduced proarrhythmia; therefore, we cannot extend our findings to these patients who experience episodes of torsade de pointes after ibutilide infusion.

The lack of any effect of ibutilide infusion on the QRS duration has been demonstrated previously, ${ }^{4,5}$ while the differences in the temporal measures of VR between cardioverted and noncardioverted patients, either before or after ibutilide infusion, should be attributed to the respective differences in heart rate between the two study groups. This study is limited by the rather small number of patients enrolled and by the fact that patients with ibutilide-induced proarrhythmia were not evaluated. Furthermore, a placebo-control group was not used in this study. However, this is the first study to present an alternative method for estimating VR alterations after ibutilide infusion. We believe that our automatic method for calculating
VCG descriptors of VR may be less vulnerable to measurement inaccuracies that are commonly faced when measuring QT intervals manually in patients with AF or AFl. More and larger studies are needed to assess the ability of this method to predict the occurrence of ibutilide-induced proarrhythmia.

\section{Conclusion}

While temporal measures of VR are significantly affected in all patients who receive ibutilide infusion for AF or AFl cardioversion, spatial VCG characteristics of VR are significantly altered only in nonresponders to the drug. A dose-related effect of ibutilide on the different aspects of VR should be suspected.

\section{Acknowledgment}

The authors are grateful to Georgia Galliou and the research nurses of the Department of Cardiology, Pammakaristos Hospital, Athens, for their valuable technical assistance.

\section{References}

1. Foster RH, Wilde MI, Markham A: Ibutilide. A review of its pharmacological properties and clinical potential in the acute management of atrial flutter and fibrillation. Drugs 1997;54:312-330

2. Jungbluth GL, VanderLugt JT, Welshman IR: Relationship between subject age and QTc interval prolongation induced by the class III antiarrhythmic drug ibutilide (abstr). Clin Pharmacol Ther 1996;59:164

3. VanderLugt JT, Gaylor SK, Wakefield LK: Effects of ibutilide fumarate, a new class III antiarrhythmic agent in man (abstr). Clin Pharmacol Ther 1991;49:188

4. Ellenbogen KA, Stambler BS, Wood MA, Sager PT, Wesley RC, Meissner MD, Zoble RG, Wakefield LK, Perry KT, VanderLugt JT: Efficacy of intravenous ibutilide for rapid termination of atrial fibrillation and atrial flutter: A dose response study. J Am Coll Cardiol 1996;28:130-136

5. Stambler BS, Wood MA, Ellenbogen KA, Perry KT, Wakefield LK, VanderLugt JT: Efficacy and safety of repeated intravenous doses of ibutilide for rapid conversion of atrial flutter or fibrillation. Circulation 1996; 94:1613-1621

6. Malik M, Acar B, Gang Y, Yap YG, Hnatkova K, Camm AJ: QT dispersion does not represent electrocardiographic interlead heterogeneity of ventricular repolarization. J Cardiovasc Electrophysiol 2000;11:835-843

7. Zabel M, Acar B, Klingenheben T, Franz MR, Hohnloser SH, Malik M: Analysis of 12-lead T-wave morphology for risk stratification after myocardial infarction. Circulation 2000;102:1252-1257

8. Dilaveris P, Gialafos E, Pantazis A, Synetos A, Triposkiadis F, Gialafos J: The spatial QRS-T angle as a marker of ventricular repolarization in hypertension. J Hum Hypertens 2001;15:63-70

9. Lee KW, Kligfield P, Dower GE, Okin PM: QT dispersion, T-wave projection, and heterogeneity of repolarization in patients with coronary artery disease. Am J Cardiol 2001;87:148-151

10. Dilaveris P, Pantazis A, Gialafos E, Triposkiadis F, Gialafos J: Determinants of electrocardiographic and spatial vectorcardiographic descriptors of ventricular repolarization in normal subjects. Am J Cardiol 2001;88:912-914

11. Dilaveris P, Pantazis A, Gialafos E, Triposkiadis F, Gialafos J: The effects of cigarette smoking on the heterogeneity of ventricular repolarization. Am Heart J 2001;142:833-837

12. Bortolan G, Bressan M, Cavaggion C, Fusaro S: Validation of QT dispersion algorithms and some clinical investigations. IEEE Computers in Cardiology 1996:665-667

13. Edenbrandt L, Houston A, Macfarlane PW: Vectorcardiograms synthesized from 12-lead ECGs: A new method applied in 1,792 children. Paediatric Cardiology 1994;15:21-26

14. Ishizawa K, Ishizawa K, Motomura M, Konishi T, Wakabayashi A: High reliability rates of spatial pattern analysis by vectorcardiogram in assessing the severity of eccentric left ventricular hypertrophy. Am Heart J 1976; 91:50-57 